\title{
Screening specific polypeptides of breast cancer stem cells from a phage display random peptide library
}

\author{
FEI LIU ${ }^{1}$, CHUN-LING QI ${ }^{2}$, MIAN KONG ${ }^{3}$, TING-TING LIU ${ }^{1}$, LEI LI ${ }^{1}$ and BAO-JIANG LI ${ }^{1}$ \\ ${ }^{1}$ Cancer Center; ${ }^{2}$ Clinical Test Center, Taian Hospital, Taishan Medical College, Taian, Shandong 271000; \\ ${ }^{3}$ Department of Thyroid Breast Surgery, The First People's Hospital of Jining, Jining, Shandong 272000, P.R. China
}

Received May 15, 2015; Accepted September 27, 2016

DOI: 10.3892/ol.2016.5248

\begin{abstract}
The present study aimed to identify polypeptides that specifically bond to breast cancer stem cells from a phage display random 12 peptide library, in addition to the affinity and specificity of polypeptides. A phage display random 12 peptide library was screened using breast cancer stem cells as targets isolated from the MDA-MB-231 cell line using the serum-free culture technique with hs578bst and MDA-MB-231 cells as subtract-screening cells. Positive and specific binding clones were amplified and sent for sequencing. The affinity and specificity of the positive clones were subsequently identified by ELISA and 3,3'-diaminobenzidine staining. The results demonstrated that phages were gathered 500 times following three rounds of biopanning. ELISA identified that the affinity to breast cancer stem cells of the no. 6 phage was 6.14 times higher than that in the control group. In addition, immunohistochemistry observed that the no. 6 phage exhibited high-specificity bonding to breast cancer stem cells, and the peptide sequence of the positive phage was GYSASRSTIPGK following DNA sequencing and translation. Thus, the present study isolated a specific peptide that bonds to breast cancer stem cells from a phage display random peptide library, which may facilitate further studies regarding the stem cell-targeted therapy of breast cancer.
\end{abstract}

\section{Introduction}

The emergence, development and drug resistance of a tumor are the results of certain characteristics of cancer stem cells. The use of targeted therapy to tumor stem cells can achieve treatment of a tumor from its origin. All types of enrichment methods for tumor stem cells (including those based on stem cell surface markers, intracellular enzyme activity,

Correspondence to: Professor Bao-Jiang Li, Cancer Center, Taian Hospital, Taishan Medical College, Taian, Shandong 271000, P.R. China

E-mail: jblrw0228@163.com

Key words: breast cancer stem cell, phage display random 12 peptide library, subtract screening, polypeptide concentration of reactive oxygen species, mitochondrial membrane potential, promoter-driven fluorescent protein expression, autofluorescence, suspension/adherent culture, cell division, identification of side population cells, resistance to cytotoxic compounds or hypoxia, invasiveness/adhesion, immunoselection and physical properties) have contributed to significant progress since Al-Hajj et al (1) isolated breast cancer stem cells successfully in 2003, thus facilitating further research on breast cancer stem cells (1). Breast cancer stem cells originate from normal mammary stem cells or differentiated breast cancer cells, which are characterized by the capability of self-renewal, differentiation and tumorigenicity (2). Breast cancer stem cells have been observed to be highly resistant to radiotherapy, chemotherapy and hormone therapy (3). Traditional radiotherapy and chemotherapy cannot easily kill breast cancer stem cells, leading to recurrence or metastasis in breast cancer. Only targeted elimination or permanent killing of breast cancer stem cells is the point at which breast cancer may be cured completely. Therefore, based on phenotypic and biological characteristics of breast cancer stem cells, research regarding technological strategy for breast cancer stem cells may benefit breast cancer treatment. The Phage Peptide Library is an efficient and simple tool that has been widely used in the development of antitumor drugs and tumor diagnostic markers (4). In the current study, breast cancer stem cells were isolated from MDA-MB-231 cells using the serum-free culture method and phage display technology was subsequently employed to screen the phages that are able to specifically bind to breast cancer stem cells.

\section{Materials and methods}

Materials. Human breast hs578bst, MDA-MB-231 and MCF-7 cell lines were obtained from the State Key Laboratory of Oncology in Southern China (Sun Yat-sen University, Guangzhou, China). Ph.D-12 Phage Display Peptide Library and its host cell (Escherichia coli; ER2738) were purchased from New England Biolabs Ltd., Hitchin, UK). Dulbecco's modified Eagle's medium(DMEM), fetal bovine serum(FBS) and trypsin were purchased from HyClone; GE Healthcare Life Sciences (Logan, UT, USA). Isopropyl $\beta$-D-1-thiogalactopyranoside was purchased from Roche Diagnostics (Basel, Switzerland) and X-gal from Sigma-Aldrich; Merck Millipore (Darmstadt, Germany). Horseradish-peroxidase (HRP)-conjugated 
anti-M13 phage antibody (27942101) was purchased from GE Healthcare Life Sciences, and ELISA staining reagents were obtained from Beyotime Institute of Biotechnology (Haimen, China). An M13 Phage Plasmid Isolation kit was purchased from Biomiga, Inc. (San Diego, CA, USA). The National Center for Biotechnology Information database (https://www.ncbi. nlm.nih.gov/) was used for bioinformatics sequence analysis.

Cell culture and isolation of breast cancer stem cells. Normal cell culture was mixed with DMEM containing 10\% FBS and penicillin-streptomycin solution under $37^{\circ} \mathrm{C}$ and $5 \%$ $\mathrm{CO}_{2}$. Medium was replaced every half day, and cytokines were added as appropriate. Epidermal growth factor, basic fibroblast growth factor and B27 were placed in serum-free medium to isolate the MDA-MB-231 breast cancer stem cells. Microsphere formation was viewed under a light microscope. Samples were centrifuged at $201 \mathrm{x} g$ for $1 \mathrm{~min}$ at room temperature, and the medium was replaced every 2 or 3 days. The cells were cultured in DMEM with $10 \%$ FBS following each generation of serum-free culture. Dead cells were removed as appropriate.

Phage screening for breast cancer stem cells. The hs578bst, MDA-MB-231 and breast cancer stem cells were isolated and cultured in poly-L-lysine-coated plates with $10^{5} /$ dish density and adherent culture. Serum-free DMEM was added for $2 \mathrm{~h}$ and the cells were sealed with $0.5 \%$ bovine serum albumin (BSA) (Beijing Solarbio Science \& Technology Co., Ltd., Beijing, China) for $1 \mathrm{~h}$. The Phage Display Peptide Library was added to the dish coated with the hs578bst cells with $10^{11} \mathrm{pfu} / \mathrm{titer}$, and the cells were cultured at $37^{\circ} \mathrm{C}$. Supernatant was subsequently added to the dishes with the MDA-MB-231 cells. Supernatant was added to the dishes with breast cancer stem cells following the same process. The supernatant was discarded after $1 \mathrm{~h}$ of incubation at $37^{\circ} \mathrm{C}$. The dishes were washed with $0.05 \%$ TBS and Tween 20 (TBST) (v/v) three times, for 1 min each time. The enriched phages were eluted with glycine elution buffer $(\mathrm{pH}, 2.2)$ and neutralized with Tris- $\mathrm{HCl}(\mathrm{pH}, 9.1)$. These phages were gathered from the first round of screening. A small amount of the eluate was obtained for amplification and used to infect its host bacteria for the next round of screening. The concentration of Tween-20 in TBST was increased to $0.1 \%(\mathrm{v} / \mathrm{v})$ and the incubation time was increased in the following experiments. Up to $10 \mu \mathrm{l}$ of the eluate was collected to measure the phage titer and compare the enrichment ratio prior to and following each screening. A total of 10 blue plaques were randomly selected at the final round of phage tittering. These phages may be used for identification and DNA sequencing following amplification.

ELISA identification of concentrated phages. The concentrated breast cancer stem cells were inoculated at a density of $10^{4} /$ hole in 96 -well plates and handled with serum-free DMEM for approximately $2 \mathrm{~h}$ following adherence. The cells were subsequently collected and fixed in $4 \%$ paraformaldehyde for 15 min, washed with PBS and disposed with $0.1 \%$ TritonX-100 for $10 \mathrm{~min}, 0.05 \%$ PBST thrice and 2\% PBS-BSA for $1 \mathrm{~h}$. The phages were added subsequent to amplification and washed with $0.05 \%$ PBST for $2 \mathrm{~h}$. HRP-conjugated anti-M13 antibody (diluted in 2\% PBS-BSA at 1:5,000) was added after
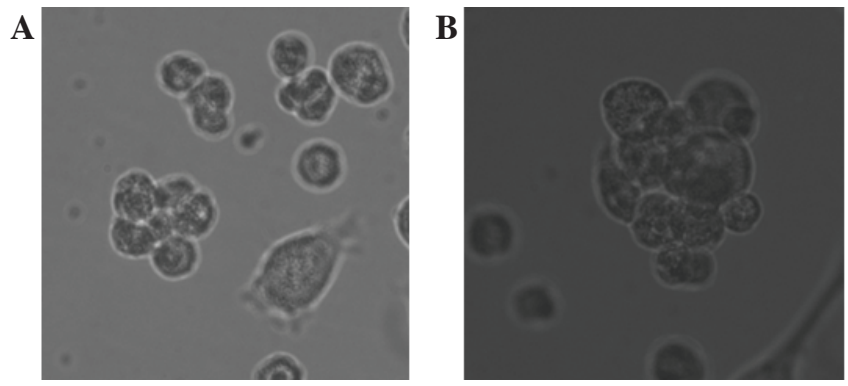

Figure 1. Breast cancer stem cell microspheres of the MDA-MB-231 cell line subsequent to suspension cultivation; (A) magnification, $\mathrm{x} 200$ and (B) x400.

$1 \mathrm{~h}$ and washed three times with $0.05 \% \mathrm{PBST}$. $\mathrm{HCl}$ was used to terminate the reaction after 3,3',5,5'-tetramethylbenzidine color development. The absorbance was measured by an ELISA microplate reader (Bio-Rad Laboratories, Inc., Hercules, CA, USA) at $450 \mathrm{~nm}$. The phages were randomly selected from the original library as a control, and if the phage optical density (OD) / control OD was $>2$, this was defined as positive.

3,3'-Diaminobenzidine (DAB) identification of concentrated phages. hs578bst, MDA-MB-231 and concentrated breast cancer stem cells were placed in 24-well plates at a density of $10^{4} /$ hole, following the steps described earlier in the 'Phage screening for breast cancer stem cells' paragraph. The differences between the two procedures were detected using one DAB chromogenic reagent kit (Beijing Dingguo Changsheng Biotechnology Co., Ltd., Beijing, China) for color development. The color reaction was inhibited with distilled water following $10 \mathrm{~min}$. Mayer's hematoxylin was used for subsequent staining and the results were observed under a light microscope.

Extraction of phage DNA and sequencing. The positive phage clone in described earlier in the 'Phage screening for breast cancer stem cells' paragraph was amplified. DNA was extracted using an M13 Phage Plasmid Isolation kit and was sent to Invitrogen (Thermo Fisher Scientific, Inc., Waltham, MA, USA) for DNA sequencing.

\section{Results}

Suspension cultivation of breast cancer stem cell microspheres. Following the serum-free culture of breast cancer cells and three rounds of 'serum-free with serum', numerous breast cancer stem cell microspheres were suspended in the cell culture medium. The microspheres increased in volume with increased incubation time and growth adhering to the wall when represented in the serum medium (Fig. 1).

Specificity phage screened for bonding to breast cancer stem cells. The phages were enriched nearly 500 times following three rounds of screening using the hs578bst and MDA-MB-231 cells as the control screen group and breast cancer stem cells as the experimental group. Screening phages decreased in degree prior to and following each round of determination (Table I). 
Table I. Screening phage drop degree prior to and following each round.

\begin{tabular}{lccc}
\hline Round & Phages in place, cpu & Phage washed out, cpu & Enrichment ratio \\
\hline First round & $1.0 \times 1011$ & $4.9 \times 103$ & $4.9 \times 10-8$ \\
Second round & $1.5 \times 1011$ & $6.5 \times 104$ & $4.3 \times 10-7$ \\
Third round & $1.5 \times 1010$ & $3.2 \times 105$ & $2.1 \times 10-5$ \\
\hline
\end{tabular}

Table II. Amino acids translated by DNA sequences for positive phages.

\begin{tabular}{ll}
\hline No. & \multicolumn{1}{c}{ Amino acids } \\
\hline P1, P7 & LPAEPPKIVKLR \\
P3, P6, P9 & GYSASRSTIPGK \\
P5 & GAIRIRLSEPLS \\
P8 & SIYVDYETNRVV \\
P10 & HSPLSAITNNIM \\
\hline
\end{tabular}

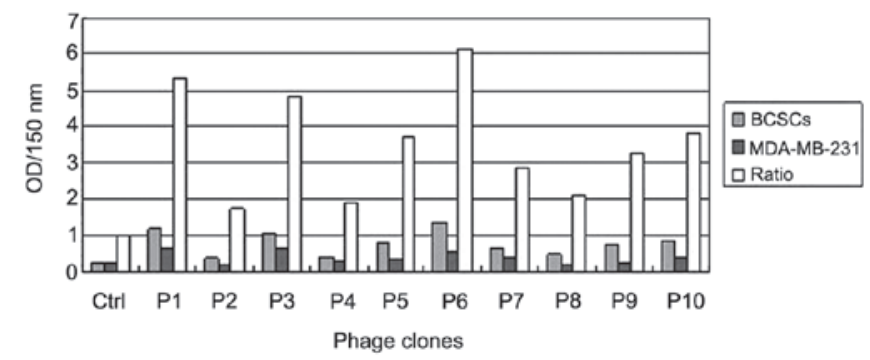

Figure 2. Identification of phage specificity to breast cancer stem cells by ELISA. OD, optical density; BCSC, breast cancer stem cell.

Identification of phage specificity to breast cancer stem cells by ELISA. A total of 10 independent phagocytic loci coerulei were selected randomly for amplification from the last round of phage titration tablet. Following three rounds of screening, the affinity of each phage to breast cancer stem cells was identified by ELISA and compared with the original library phage that was randomly selected (Fig. 2). Among the phages, no. 6 exhibited the highest affinity to target cells and was labeled KL-6.

$D A B$ identification of phage bonding and specificity to breast cancer stem cells. DAB staining was used to identify the specificity of the positive phage clone KL- 6 to breast cancer stem cells (Fig. 3). KL-6 exhibited the highest affinity and specificity to the breast cancer stem cells, which were clearly stained brown. KL-6 demonstrated small affinity and specificity to the MDA-MB-231 breast cancer cells, with a few partially stained cells, and exhibited no affinity or specificity to the MCF-7 breast cancer cells and breast cells, in which no stained cells were identified.

DNA sequencing for positive phages. Following ELISA identification and amplification, 8 positive phages were collected by M13 Phage Plasmid Isolation kit. Approximately
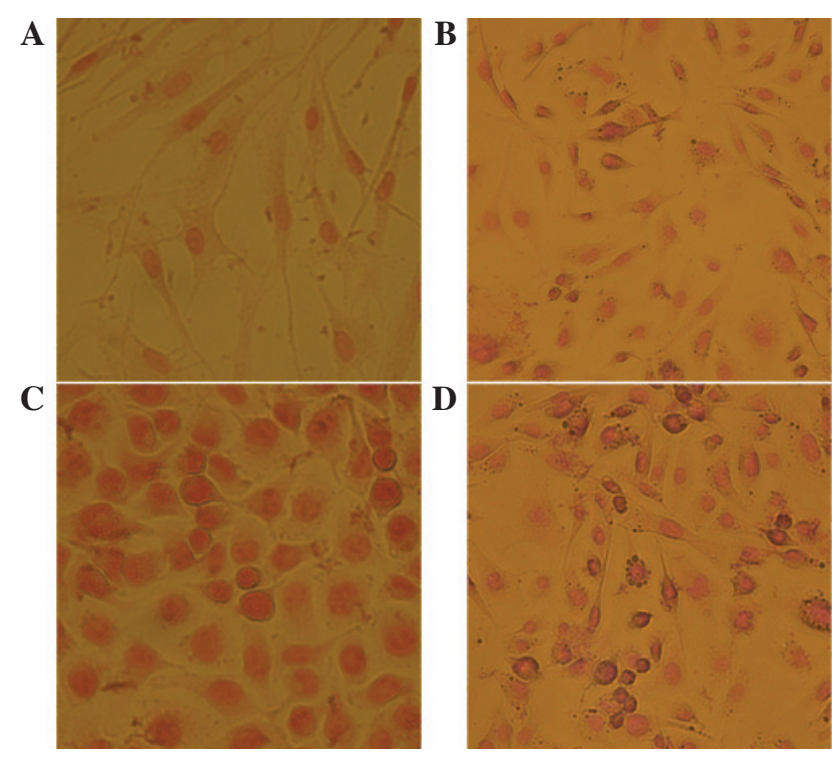

Figure 3. 3,3'-Diaminobenzidine stain for positive phages in the (A) hs578bst, (B) MDA-MB-231, (C) MCF-7 and (D) breast cancer stem cells (magnification, $\mathrm{x} 200)$.

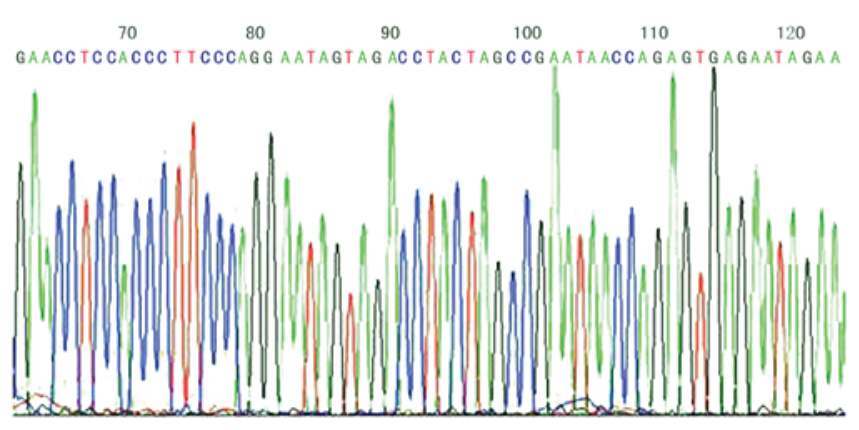

Figure 4. DNA sequencing of the KL-6 phage.

$5 \mu \mathrm{g}$ DNA was obtained from $3 \mathrm{ml}$ phage incubation buffer. The amino acids translated by DNA sequences are presented in Table II, in which a sequence (GYSASRSTIPGK) appeared thrice; this sequence is unknown when compared with the Basic Local Alignment Search Tool (http://www.ncbi.nlm.nih. gov/Blast). The DNA sequencing of KL-6 phage 12 peptide fragments is illustrated in Fig. 4.

\section{Discussion}

Reya et al (5) proposed the 'theory of tumor stem cells' in 2001, which suggested that not all cells in tumor tissues are able to proliferate aberrantly and eventually become malignant. However, a few cancer stem cells do control the development, 
transfer and drug resistance of tumors, which primarily cause tumorigenesis and recurrence (6). A number of experiments and studies have been conducted on cancer stem cells. Based on the existing stem cell separation technology, the association between breast cancer stem cells and viruses, expression of estrogen and radiation treatment have been studied (7-9). In 2012, three separate teams of researchers used genetic marker technology to track cell proliferation, particularly cancer cell growth, which provided strong evidence to confirm the existence of cancer stem cells (10-12). Surgery, chemotherapy, radiotherapy and targeted therapies are currently the most common therapeutic methods for breast cancer. The targeted therapy of stem cells also exhibits good prospects for development and considerable economic potentials (13). The development of targeted peptides for breast cancer stem cells may reduce drug dosage and side effects of chemotherapy drugs on normal cells. Breast cancer may also be cured radically in this approach.

Phage display is able to obtain target specificity phage clones without immunization in a short time (14). The screening procedure is simple and extremely useful in the field, such as in antigen epitope, parasites and tumor-specific marker screening $(15,16)$. Gur et al (17) employed phage display technology to screen two polypeptides for SUM159 breast cancer stem cells. These cells may be targeted to identify breast cancer stem cells. Phage display technology is used in screening the specificity and polypeptide of tumor stem cells, which has provided novel potential approaches for breast cancer treatment (18).

Cluster of differentiation (CD)133 is one of the numerous markers used in screening targets for cancer stem cells. However, the roles of CD133 and its natural ligand in breast cancer stem cells remain unknown. Sun et al (19) employed phage display technology and obtained a peptide sequence (LQNAPRS) that is able to specifically bind to CD133 in nude mice. Migration and wound healing experiments indicated that this peptide may inhibit the migration of breast cancer cells through concentration dependence (19). In addition, Tian et al (20) also obtained one phage peptide using similar experiments and methods, and this phage peptide may also specifically combine with CD133 and simulate the molecular ligands and antigen epitope of human colon cancer, which is expected to develop peptide drugs via antagonism effect. Furthermore, Yang et al (21) detected a sequence via Phage Peptide Library screening for NVVRQ five peptides, and this peptide is able to bind to highly metastatic tumor cells, including MDA-MB-435 breast cancer cells specifically in vivo and in vitro.

The current study also designed dual-subtract biopanning, which is a method that is more rigorous than conventional screening methods in the process of experimental screening. This method is able to address the shortcomings of serum-free culture method, but cannot enrich all the stem cells in it. The serum-free culture method was employed by the present study in the steps in enrichment of breast cancer stem cells as this method causes minimal damage to the stem cells. This method avoided the disadvantages of the side-group cell separation method, including surface antigen changes on stem cells (22). The one-sidedness of the separation method was also resolved using CD $44^{+} / \mathrm{CD} 24^{-}$as a cell-surface marker. This method does not only enrich stem cells without the change of surface antigens, but also ensures the diversity of specific antigens on the surface of stem cells. Given that no method is able to fully select the stem cells, enough positive phages can be screened by the dual-subtract biopanning method. The phage-specific evaluation process itself is also the appraisal process of sorting out the stem cell population. In the current study, the DNA sequence of the phage was transferred into an amino acid (GYSASRSTIPGK) following extraction and compared with the National Center for Biotechnology Information GenBank DNA database sequence of bioinformatics analysis (https:// www.ncbi.nlm.nih.gov/). The results demonstrated that the phage peptide sequence was unknown, and no homology with genes and proteins were known. Thus, it may be speculated that the present study screened one novel surface antigen-related ligand for breast cancer stem cells using phage display technology and facilitated further research on stem cell biology and new breast cancer-targeted therapy.

Cancer stem cells have elicited considerable attention in the field of cancer research. However, previous findings are not established enough to guide clinical treatment and direct further effective breast cancer treatment. The current study used a serum-free medium suspension culture method to obtain specific polypeptide sequence binding to breast cancer stem cells using phage display technology. As expected, a novel chemotherapy drug targeted to breast cancer stem cells was developed using these peptides as carriers, and this new drug may exhibit wider application than existing drugs, including Herceptin, for curing breast cancer from its origin. Simultaneously, this technology may be applied to other breast cancer cell lines to enrich the stem cells and use breast tissues in animal experiments in vivo to validate the specificity of the polypeptide. Further research is also necessary for the application of the current results in clinical treatment.

In conclusion, breast cancer stem cells were successfully enriched using serum-free suspension culture method in the present study. Additionally, polypeptides that specifically bound to breast cancer stem cells were identified from a phage display random 12 peptide library. Future studies will be conducted to identify specific surface antigens of breast cancer stem cells. These results may provide a foundation and mechanism-based guide for targeted breast cancer therapies.

\section{References}

1. Al-Hajj M, Wicha MS, Benito-Hernandez A, Morrison SJ and Clarke MF: Prospective identification of tumorigenic breast cancer cells. Proc Natl Acad Sci USA 100: 3983-3988, 2003.

2. Kai K, Arima Y, Kamiya T and Saya H: Breast cancer stem cells. Breast Cancer 17: 80-85, 2010.

3. Calcagno AM, Salcido CD, Gillet JP, Wu CP, Fostel JM, Mumau MD, Gottesman MM, Varticovski L and Ambudkar SV: Prolonged drug selection of breast cancer cells and enrichment of cancer stem cell characteristics. J Natl Cancer Inst 102: 1637-1652, 2010.

4. Du B, Qian M, Zhou Z, Wang P, Wang L, Zhang X, Wu M, Zhang $\mathrm{P}$ and Mei B: In vitro panning of a targeting peptide to hepatocarcinoma from a phage display peptide library. Biochem Biophys Res Commun 342: 956-962, 2006.

5. Reya T, Morrison SJ, Clarke MF and Weissman IL: Stem cells, cancer, and cancer stem cells. Nature 414: 105-111, 2001.

6. Gangopadhyay S, Nandy A, Hor P and Mukhopadhyay A: Breast cancer stem cells: A novel therapeutic target. Clin Breast Cancer 13: 7-15, 2013.

7. Li J, Zeng W, Huang Y, Zhang Q, Hu P, Rabkin SD and Liu R: Treatment of breast cancer stem cells with oncolytic herpes simplex virus. Cancer Gene Ther 19: 707-714, 2012. 
8. Simões BM, Piva M, Iriondo O, Comaills V, López-Ruiz JA Zabalza I, Mieza JA, Acinas O and Vivanco MD: Effects of estrogen on the proportion of stem cells in the breast. Breast Cancer Res Treat 129: 23-35, 2011.

9. Zielske SP, Spalding AC, Wicha MS and Lawrence TS: Ablation of breast cancer stem cells with radiation. Transl Oncol 4: 227-233, 2011.

10. Driessens G, Beck B, Caauwe A, Simons BD and Blanpain C: Defining the mode of tumour growth by clonal analysis. Nature 488: 527-530, 2012.

11. Schepers AG, Hugo HJ, Stange DE, van den Born M, van Es JH, van de Wetering $M$ and Clevers H: Lineage tracing reveals Lgr5 stem cell activity in mouse intestinal adenomas. Science 337: 730-735, 2012.

12. Chen J, Li Y, Yu TS, McKay RM, Burns DK, Kernie SG and Parada LF: A restricted cell population propagates glioblastoma growth after chemotherapy. Nature 488: 522-526, 2012.

13. Lou H and Dean M: Targeted therapy for cancer stem cells: The patched pathway and ABC transporters. Oncogene 26: 1357-1360, 2007.

14. Hoogenboom HR and Chames P: Natural and designer binding sites made by phage display technology. Immunol Today 21 : 371-378, 2000 .

15. Guo AJ and Cai XP: Application of filamentous phage display technology in parasitology. Zhongguo Ji Sheng Chong Xue Yu Ji Sheng Chong Bing Za Zhi 24: 304-308, 2006 (In Chinese).
16. Yip YL and Ward RL: Application of phage display technology to cancer research. Currt Pharm Biotechnol 3: 29-43, 2002

17. Gur D, Liu S, Shukla A, Pero SC, Wicha MS and Krag DN: Identification of single chain antibodies to breast cancer stem cells using phage display. Biotechnol Prog 25: 1780-1787, 2009.

18. Staquicini FI, Sidman RL, Arap W and Pasqualini R: Phage display technology for stem cell delivery and systemic therapy. Adv Drug Deliv Rev 62: 1213-1216, 2010.

19. Sun J, Zhang C, Liu G, Liu H, Zhou C, Lu Y, Zhou C, Yuan L and Li X: A novel mouse CD133 binding-peptide screened by phage display inhibits cancer cell motility in vitro. Clin Exp Metastasis 29: 185-196, 2012.

20. Tian PG, Zhou CP, Zhang C, Yang H, Wu XJ, Lu YX, Liu GB and Li XN: Selection and identification of specific binding peptides for cancer stem cell surface marker CD133. Nan Fang Yi Ke Da Xue Xue Bao 31: 761-766, 2011 (In Chinese).

21. Yang W, Luo D, Wang S, Wang R, Chen R, Liu Y, Zhu T, Ma X, Liu R, Xu G, et al: TMTP1, a novel tumor-homing peptide specifically targeting metastasis. Clin Cancer Res 14: 5494-5502, 2008.

22. Liu ZZ, Chen P, Lu ZD, Cui SD and Dong ZM: Enrichment of breast cancer stem cells using a keratinocyte serum-free medium. Chin Med J (Engl) 124: 2934-2936, 2011. 